leur diamètre dans des dimensions comprises entre 80 et $120 \mathrm{~m} \mu$.

Les mesures des vitesses de sédimentation du phosphocaséinate de calcium colloïdal effectuées la même année, font constater également à Ford et RAMSDELL [17], la forme sphérique des particules.

En 1951 enfin, Hostettler et Imноғ [26] qui, employant la technique électronique, concluent dans le même sens, affirment en outre que le degré de dispersion des particules de caséinate natif est très variable d'un individu à l'autre et qu'il existe des différences sensibles entre les laits des diverses espèces animales; les micelles d'un lait de chèvre sont plus grossièrement dispersées que celles d'un lait de vache, alors que celles d'un lait de femme sont finement divisées. Le degré de dispersion du caséinate natif peut être modifié en faveur de petites particules, sous l'effet de troubles organiques généraux ou de maladies de la glande mammaire. On ne sait par contre pas actuellement dans quelle mesure la race, le stade de lactation et l'état de nutrition du sujet, sont susceptibles d'influer sur l'état de division des micelles de caséine dans le lait.

(A suivre.)

\title{
"OVERRUN": L'ARTËRE VITALE DE LA BEURRERIE CANADIENNE
}

\author{
par \\ A. C. F. KRUIJER
}

\section{INTRODUCTION}

De nos observations et travaux dans les beurreries canadiennes nous voulons présenter quelques impressions risquant d'être utiles à des industries laitières françaises.

Le principe de la fabrication du beurre ne diffère guère de la méthode européẹnne : refroidir la crème et la garder suffisamment longtemps à une température basse avant le barattage. Comme nous le savons, ce traitement thermique est d'importance, pour l'obtention d'un beurre de bonne consistance, laissant un minimum de matière grasse dans le babeurre.

Des complications surgissent, dûes aux conditions pénibles de ramassage de la crème sur de grandes distances $(200 \mathrm{~km}$. et. plus n'est pas extraordinaire). La crème arrivant souvent à l'état acide, doit être neutralisée (par le bicarbonate de soude à une température de $35^{\circ}$ C.), avant la pasteurisation, exigée par la loi, qui s'effectue généralement à $85^{\circ} \mathrm{C}$. pendant quelques (!) minutes.

On distingue trois genres de beurres : "cultured butter", 
fabriqué à partir de la crème à maturité; "swett butter ", non salé, fabriqué avec de la crème douce (neutralisée), et “butter », salé ( 1,5 à $3,0 \%$ de sel), également obtenu à partir de la crème douce. Le "cultured butter" est vendu dans la province de Québec, les autres beurres dans le reste du Canada. "Sweet butter » est surtout absorbé par les industries de crème glacée.

Le Vacreator, pasteurisateur-désodorisateur, a une certaine utilité dans ce pays où l'état de la erème laisse souvent beaucoup à désirer. Celle-ci est payée d'après sa teneur en matière grasse et ses qualités organoleptiques. La mauvaise crème coûte environ 55 francs de moins que la bonne par livre de matière grasse; si l'on peut en tirer du beurre de première classe au moyen d'un vacreator, l'installation coûteuse - de frais d'opérations élevées, laissant un babeurre de haute teneur en matière grasse - peut quand même être rentable. Il reste à considérer qu'il est souvent nécessaire de "vacréer " plusieurs fois la même crème avant d'être certain d'en pouvoir faire du bon beurre, et ensuite, que toute la quantité de crème reçue, celle de bonne qualité inclue, doit subir ces opérations. Nous ne disposons pas d'assez de chiffres pour nous permettre de calculer jusqu'à quel point le vacréator peut être rentable; nous sommes pourtant capables de constater que seulement dans le cas où la grande majorité de crème reçue est de mauvaise qualité (un minimum de $80 \%$ ne nous semble certainement pas exagéré), le vacréator est économiquement justifiable.

\section{Overrun}

Dans la beurrerie, l'overrun est le surplus de kilogramme de beurre obtenu à partir de 100 kilogrammes de matière grasse reçue.

Si la loi exige que le beurre contienne $80 \%$ de matière grasse, il s'en suit que théoriquement on pourrait fabriquer 125 kilogrammes de beurre avec 100 kilogrammes de matière grasse, soit que l'" overrun " ou surplus est $25 \%$.

Le surplus pratique est encore déterminé par d'autres facteurs : perte de matière grasse par le babeurre, limite imposée à la teneur en eau du beurre $(16,0 \%)$, teneur en sel et en "restes" - constituants (protéides, caséine complex, lactose, lactates et minéraux).

La perte en matière grasse par le babeurre dépend en grande partie de la teneur en matière grasse de la crème barattée. Plus la crème est riche, moins la quantité absolue de matière grasse dans le babeurre est grande, bien que relativement, en pourcentage, la teneur en matière grasse du babeurre est moins élevée à partir d'une crème maigre.

Au Canada, on essaie d'entraîner le producteur à livrer de la crème à $35 \%$ de matière grasse. Au cours de nos travaux, cette 
teneur de la crème à baratter, variait de 29 à $33 \%$, avec une moyenne de $32 \%$. L'avantage d'une crème riche est évident vu les frais de transport et de pasteurisation, d'autant plus que le babeurre n'a pas de valeur substantielle.

Pour en revenir à l' overrun" ou "surplus ", il faut savoir de quelle manière on détermine dans la beurrerie, la quantité de matière grasse reçue : la crème de chaque bidon est pesée, un prélèvement en est tiré, contrôlé sur des qualités organoleptiques et journellement analysé sur sa teneur en matière grasse par la méthode Babcock, teneur donnée avec une précision d'une unité (la méthode n'étant pas plus exacte, et même pas entièrement exacte pour cela).

Chaque jour, on connaît donc la quantité totale de matière grasse reçue et le lendemain, on contrôlera combien de beurre en a été tiré, puis le surplus est calculé. Ce contrôle sur le surplus ne peut pas facilement être effectué tous les jours, mais on pourra toujours s'arranger pour être fixé toutes les semaines.

Le beurrier, de son côté, contrôle le surplus de chaque barattage : il mesure la quantité de crème à baratter et en fait analyser la teneur en matière grasse.

Sur quel surplus faut-il compter? Si l'expérience apprend que le beurre obtenu est constitué en dehors de la matière grasse de la façon suivante : sel, $2,0 \%$; eau, $16 \%$; restes constituants, $1,0 \%$, et si on tolère $1,0 \%$ de perte de matière grasse par le babeurre, on arrive à une équation, basée sur le fait que la somme de matière grasse de départ (100) et du surplus (S), est égale à la somme des constituants du beurre obtenu :

$$
\begin{gathered}
(100+\mathrm{S})=(100-1,0 / 100 \times 100)+\frac{(2,0+16,0+1,0)}{100} \times(100+\mathrm{S}) \\
\mathrm{S}=22,2
\end{gathered}
$$

Dans la pratique, S est exprimé en poureentage.

Les variations du surplus indiquent des négligences dans au moins une des opérations : pesage, prélèvement de la crème, pertes dues aux manipulations de la crème, ou bien travail du beurrier.

Ce dernier a le surplus de chaque barattage sous contrôle : il connaît la quantité de matière grasse qu'il va baratter et c'est à lui d'en faire du beurre qui contienne le maximum tolérable en eau et en sel.

Sans entrer en détails sur la constitution du beurre canadien, nous allons voir quelles conclusions seront utiles en France.

D'abord le beurre doux - tiré de la crème douce - ne se fabrique pas en quantités importantes. Pour le beurre fait de crème à maturité, il est à conseiller de ne pas le saler. Il reste donc dans nos 
considérations, le beurre aromatique non-salé, beurre constitué de matière grasse, eau et restes-constituants.

La teneur en restes-constituants sera en moyenne, $1,1 \%$, celle en eau ne doit pas dépasser $16,0 \%$, tandis que la perte en matière grasse par le babeurre peut être 1,0\%. Cette perte diminuera, si la teneur en matière grasse de la crème augmente. Nous pensons alors, également du point de vue économique, qu'il est recommandable de partir d'une crème riche (30 à $35 \%$ de matière grasse). Ces données nous permettent de calculer, de façon analogue à celle décrite ci-dessus, le surplus théorique, et nous trouvons : $\mathrm{S}=19,4 \%$.

Si cela nous semble haut par comparaison au surplus calculé par d'autres méthodes, afin de prévoir la quantité de beurre, nous devons réaliser que les autres méthodes sont basées sur des moyennes de matière grasse du beurre (environ $83,5 \%$ ), tandis que le calcul du surplus est basé sur les constituants non-gras, ce qui laisse dans notre cas, une teneur de $82,9 \%$ de matière grasse dans le beurre. Si cette différence de $0,6 \%$ ne semble peut-être pas importante, en pratique cela signifie qu'une usine transformant 150.000 kilogrammes de matière grasse par an, obtiendra 1.300 kilogrammes de beurre en plus, si celui-ci contient $82,9 \%$ de matière grasse, que s'il en contient $83,5 \%$; différence payant, au prix où en est le beurre, deux à trois fois le salaire d'un beurrier.

L'importance du surplus étant évidente, il nous faut savoir comment l'exploiter. Dans les calculs qui suivent, nous partirons d'un surplus de $19,4 \%$, basé alors sur une perte de matière grasse par le babeurre de $1,0 \%$, et sur une teneur en restes-constituants de $1,1 \%$. Chaque beurrerie est capable d'analyser ces deux valeurs; si la première est inférieure à $1,0 \%$, il n'y a rien de plus à exiger ; si elle est supérieure, il faut faire le nécessaire pour l'abaisser. (Quelques facteurs à dominer : temps et température à laquelle la crème a été gardée avant le barattage; traitement du lait et de la crème à la ferme; température de l'écrémage, manipulations plus ou moins vigoureuses homogénéisant partiellement lait et crème, remplissage de la baratte.) Si la teneur en restes-constituants est trop basse, on surveillera de plus près le degré d'acidité de la crème prête à baratter et la quantité d'eau servant au lavage du beurre.

\section{Calculs}

Si on part de 2.500 kilogrammes de crème, d'une teneur en matière grasse de $33,5 \%$, on disposera de 838 kilogrammes de matière grasse et $-\mathrm{S}$ étant $19,4 \%-\frac{119,4}{100} \times 838$ soit $1.000 \mathrm{~kg}$. de beurre sont à prévoir.

Le beurrier ayant obtenu un beurre presque fini, en prendra 
un prélèvement pour l'analyser sur sa teneur en eau; il trouvera par exemple, $14,6 \%$. La question qui se pose maintenant est : combien d'eau faut-il verser dans la baratte et incorporer au beurre pour donner à ce dernier une teneur de $16,0 \%$ en eau ?

Pour bien dominer le problème, il faut réaliser qu'à ce moment, il se trouve dans la baratte (quantité d'eau supposée E) :

$$
\begin{aligned}
& \text { Kilogr. } \\
& \text { Matière grasse }\{838-1 / 100 \times 838) .=829,6 \\
& \text { Restes-constituants }(1,1 / 100 \times 1.000)=11,0 \\
& \text { Eau .................... } E \\
& \text { Total.............. } \quad(840, \overline{6}+\mathrm{E})
\end{aligned}
$$

Sachant que le prélèvement contient $14,6 \%$ en eau, l'équation suivante nous fait connaître la valeur de $\mathrm{E}$ :

$$
\begin{aligned}
& \mathrm{E}=14,6 / 100 \times(840,6+\mathrm{E}) \\
& \mathrm{E}=144 \mathrm{~kg} .
\end{aligned}
$$

Nous voulons atteindre $16,0 \%$ de 1.000 kilogrammes, soit 160 kilogrammes d'eau, done il faudra ajouter $(160-144)$, soit 16 kilogrammes d'eau.

Il est plus facile mais moins exact, de raisonner ainsi : nous trouvons $14,6 \%$ d'eau, nous en voulons $16,0 \%$; nous ajoutons done $(16,0-14,6)$, soit $1,4 \%$ de $1.000 \mathrm{~kg}$. soit $14 \mathrm{~kg}$. d'eau. Nous trouvons ainsi une quantité inférieure (de $2 \mathrm{~kg}$.), due au fait que dans le dernier calcul, on a sous-entendu que le $14,6 \%$ valait pour $1.000 \mathrm{~kg}$., alors qu'en réalité, e'était seulement pour $1.000 \mathrm{~kg}$., moins la quantité d'eau à ajouter.

\section{Pratique}

Dans la pratique, le calcul simplifié a de la valeur : il est plus prudent de rester légèrement au-dessous de la limite tolérée et le travail du beurrier est plus simple (la teneur en eau dans notre cas sera ainsi $15,8 \%$ ).

Pour lui, les calculs seront alors réduits à quelques multiplications : teneur en matière grasse de la crème $(m) \times$ un centième de la quantité de crème $(q) \times \frac{(100+S)}{100}$ est égal à la quantité estimée de beurre (B) :

$$
\mathrm{B}=\mathrm{m} \cdot \mathrm{q} \cdot \frac{100+\mathrm{S}}{100}
$$

et ensuite, pour savoir combien d'eau (E) il faut ajouter, il devra 
multiplier la différence des teneurs en eau désirée $\left(e_{d}\right)$ et trouvée $\left(e_{t}\right)$ par un centième de la quantité estimée de beurre :

$$
E=\left(e_{d}-e_{l}\right) \frac{B}{100}
$$

Chaque beurrier est capable de connaître la quantité de crème et faire analyser sa teneur en matière grasse; avec des appareils simples comme on les trouve chez 'les fournisseurs d'industries laitières, l'analyse de la teneur en eau ne présente aucune difficulté.

Pour rendre l'exécution encore plus simple, le directeur de laiterie fera une table couvrant les valeurs les plus courantes dans son affaire, et la mettra à la disposition du beurrier.

La chose principale est que le directeur de la beurrerie contrôle ou fasse contrôler la valeur du surplus S, ce qui veut dire qu'il doit connaître la perte en matière grasse par le babeurre, et la teneur en restes-constituants du beurre.

\section{Conclusions}

Le rendement en beurre est un facteur de première importance dans la beurrerie ou dans toutes les industries laitières dans lesquelles la fabrication du beurre fait partie.

Un contrôle périodique du surplus effectue un contrôle direct sur les opérations.

La connaissance du surplus aide à pouvoir obtenir un rendement maximum en beurre.

L'art du beurrier - chaque barattage de bonnes consistance et structure demande de l'art beurrier! - n'est restreint en aucune façon, au contraire s'enrichit : il faut que le beurrier sache choisir le bon moment pour prendre le prélèvement - d'un beurre pas entièrement fini de travailler, dans une baratte qui est humide, mais qui ne montre pas de larges quantités d'eau (flaques) - puis bien faire incorporer l'eau ajoutée au beurre sans le surtravailler. 